INPLASY

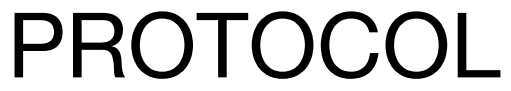

To cite: Guo et al. Association of sarcopenia with falls and fractures in the elderly : an updated systematic review and meta-analysis. Inplasy protocol 2021110030. doi:

10.37766/inplasy2021.11.0030

Received: 09 November 2021

Published: 09 November 2021

Corresponding author:

Siyuan Guo

guosy@tit.edu.cn

Author Affiliation:

Jeonju University.

Support: All funds come from the author.

Review Stage at time of this submission: Preliminary

searches.

Conflicts of interest:

None declared.

\section{Association of sarcopenia with falls and fractures in the elderly : an updated systematic review and meta-analysis}

Guo, Syㅜ; Sohn, Jh².

Review question / Objective: Mean or median age of $\geq 65$ years or with subgroup analysis in those aged $\geq 65$ years; diagnosis of sarcopenia using any definition used by the cut-off values of decreased muscle mass and/or decreased muscle strength and/or decreased physical ability; and at least one of the following outcomes: falls and/or fractures.The research questions are as follows: Association between sarcopenia and falls in older adults. Association between sarcopenia and fractures in older adults. Association between sarcopenia and risk of falls and fractures in older adults.

Information sources: We will search the following electronic bibliographic databases: MEDLINE, EMBASE, Cochrane, Web of science and CINAHL. The search strategy will include terms relating to or describing sarcopenia, falls and fractures. Only studies written in English will be included. All studies until the date of the last search $(28 / 10 / 21)$ will be sought.

INPLASY registration number: This protocol was registered with the International Platform of Registered Systematic Review and Meta-Analysis Protocols (INPLASY) on 09 November 2021 and was last updated on 09 November 2021 (registration number INPLASY2021110030).

\section{INTRODUCTION}

Review question / Objective: Mean or median age of $\geq 65$ years or with subgroup analysis in those aged $\geq 65$ years; diagnosis of sarcopenia using any definition used by the cut-off values of decreased muscle mass and/or decreased muscle strength and/or decreased physical ability; and at least one of the following outcomes: falls and/or fractures. The research questions are as follows: Association between sarcopenia and falls in older adults. Association between sarcopenia and 
fractures in older adults. Association between sarcopenia and risk of falls and fractures in older adults.

Condition being studied: Association of sarcopenia with falls and fractures in the elderly. Titles and/or abstracts of studies retrieved using the search strategy and from the reference lists of systematic review will be screened independently by two review authors to identify studies that potentially meet the inclusion criteria outlined above. The full text of these potentially eligible studies will be retrieved and independently assessed for eligibility by two review authors. Any disagreement between them over the eligibility of particular studies will be resolved through discussion with a third review author. A standardized, pre-piloted form will be used to extract data from the included studies for assessment of study quality and evidence synthesis. Extracted information will include: author; year of publication; study design; setting; sample size; age of participants; percentage of females/males; definition of sarcopenia; prevalence of sarcopenia; assessment method of bone mineral density, muscle mass and muscle strength; fall outcomes with follow-up period; method of fall assessment (e.g. falls calendar, telephone interview); method of fracture assessment (e.g. questionnaire, radiographic report); type of fracture; proportion of fallers by non-sarcopenia and sarcopenia subgroup; proportion of participants with fracture by nonsarcopenia and sarcopenia subgroup; risk of bias. Two authors will independently assess risk of bias, discrepancies will be identified and resolved through discussion (with a third author where necessary). Missing data will be requested from study authors.

\section{METHODS}

Participant or population: Inclusion criteria: older adults (mean age or median 65 years and above); all definitions of sarcopenia; at least one of the following outcomes is assessed: falls and/or fractures; English language; no restriction on disease group of the study population.Exclusion criteria: none.

Intervention: Participants with sarcopenia who do/do not experience falls and/or fractures will be compared to participants without sarcopenia who do/do not experience falls and/or fractures.

Comparator: Sarcopenia as defined by any definitions using muscle mass alone, and definitions that combine muscle mass with muscle strength and/or physical performance.

Study designs to be included: Case-control studies, cross-sectional studies, cohort studies and baseline measurements of randomised controlled trials will be included. Inclusion criteria: older adults with mean/median age equal or above 65 years, all definitions of sarcopenia; at least one of the following outcomes is assessed: falls and/or fractures; English language; no restriction on disease group of the study population. Exclusion: studies without primary data such as conference abstracts; reviews; editorials; letters to the editor; case reports with fewer than 5 cases.

Eligibility criteria: Mean or median age of $\geq 65$ years or with subgroup analysis in those aged $\geq 65$ years; diagnosis of osteosarcopenia using any definition used by the cut-off values of decreased muscle mass and/or decreased muscle strength and/or decreased physical ability; and at least one of the following outcomes: falls and/or fractures.

Information sources: We will search the following electronic bibliographic databases: MEDLINE, EMBASE, Cochrane, Web of science and CINAHL. The search strategy will include terms relating to or describing sarcopenia, falls and fractures. Only studies written in English will be included. All studies until the date of the last search (28/10/21) will be sought.

Main outcome(s): Falls (Yes/No), fractures (Yes/No), risk of falls and fractures (category or score). 
Quality assessment / Risk of bias analysis: Two authors will independently assess the risk of bias by extracting the following information: (1) characteristics of study cohort (age, sex, study population, setting), (2) assessment of outcome in case of: a) sarcopenia: definition used, methods used to assess diagnostic criteria; b) falls assessment: method used, interval of falls assessment, follow-up period; c) fracture assessment: method used, type of fracture: method used, type of fracture, (3) description of outcome: outcome data reported with odds ratios/risk ratios, $95 \%$ confidence interval and p-value given, (4) adjustment for confounding variables (age, sex, multimorbidity, BMI, Bone mineral density).

Strategy of data synthesis: We will make a narrative synthesis of the results of the included studies. The extracted data will be divided into falls or fractures and stratified by setting (community, nursing home/hotel and hospital). We will provide a summary for each study by calculating the odds ratio or hazard ratio (for dichotomous results) or standardized average difference (for consecutive secondary results). A metaanalysis will be performed to estimate: falls or fractures in participants with or without sarcopenia. The analysis will be stratified by environment (community, nursing home/ hotel and hospital) and age. The I-square statistic will be used to assess the heterogeneity of the study in effect measurement. We will consider I-square values greater than $50 \%$ to indicate significant heterogeneity. If the number of included studies permits, we will use a stratified meta-analysis to explore the heterogeneity of effect estimates based on the following factors: study quality; environment; definition of sarcopenia; measures of fall, and body mass index. We will also evaluate the evidence for publication bias.

Subgroup analysis: Subgroup analysis will be conducted when sufficient numbers of studies are identified for: setting/ population, different sarcopenia definitions and body mass index.
Sensitivity analysis: Odds ratio's and $95 \%$ confidence intervals.

Country(ies) involved: South Korea.

Keywords: sarcopenia; falls; fractures; elderly.

Contributions of each author:

Author 1 - Siyuan Guo - Find literature and extract data. Writing the whole article.

Email: guosy@tit.edu.cn

Author 2 - Jeehoon Sohn - Review the literature and make an overall assessment of the article. Evaluation of sources of heterogeneity. 